\title{
Downregulation of inhibitor of growth 3 is correlated with tumorigenesis and progression of hepatocellular carcinoma
}

\author{
MEILING LU ${ }^{1,2}$, FEI CHEN ${ }^{2}$, QINWAN WANG ${ }^{1,2}$, KESHENG WANG $^{1,2}$, QIUHUI PAN ${ }^{1}$ and XIN ZHANG ${ }^{2}$ \\ ${ }^{1}$ The Central Laboratory, People's 10th Hospital, Shanghai 200072; \\ ${ }^{2}$ Chinese National Human Genome Center at Shanghai, Shanghai 201203, P.R. China
}

Received January 31, 2012; Accepted March 30, 2012

DOI: $10.3892 / \mathrm{ol} .2012 .685$

\begin{abstract}
ING3, a member of the inhibitor of growth (ING) family, has been reported to be involved in transcription modulation, cell cycle control and the induction of apoptosis. Previous studies have demonstrated that the expression of ING3 decreased in melanoma and head and neck squamous cell carcinoma (HNSCC). The aim of this study was to investigate the role of ING3 in hepatocellular carcinoma (HCC) tumorigenesis and progression. The correlation between ING3 expression and clinicopathological variables of HCC was analyzed. Using the real-time reverse transcription-polymerase chain reaction (RT-PCR), it was found that ING3 was downregulated in HCC tissues compared with adjacent non-cancerous tissues $(\mathrm{p}<0.05)$. The immunohistochemical staining of tissue microarray data indicated a significant reduction of ING3 expression in $57.14 \%$ of HCC cases (64/112). In addition, the downregulation of ING3 was associated with the tumor differentiation stage. Most HCC samples of Edmondson-Steiner grades II to III exhibited inhibition of ING3 expression. The overexpression of ING3 in HCC cells was found to suppress cell proliferation, colony formation and cell migration, suggesting that ING3 acts as a tumor suppressor in HCC cells. Taken together, the data revealed that ING3 may serve as a suppression factor during tumorigenesis and progression of HCC.
\end{abstract}

\section{Introduction}

The inhibitor of growth (ING) family of type II tumor suppressors comprises five conserved genes, ING1-5, which share $32-76 \%$ DNA sequence homology (1-7). ING proteins contain a conserved plant homeodomain (PHD) in the C-terminal region, a nuclear localization signal (NLS) in the middle region and a novel conserved region (NCR) of unknown function at the N-terminus (8). Results of previous studies showed that

Correspondence to: $\mathrm{Dr}$ Xin Zhang, Chinese National Human Genome Center at Shanghai, 351 Guo Shou-Jing Road, Shanghai 201203, P.R. China

E-mail: zhang68@hotmail.com

Key words: ING3, tumorigenesis, hepatocellular carcinoma, cell proliferation, cell migration
ING genes are involved in DNA repair, chromatin remodeling, cell cycle control, senescence and apoptosis $(9,10)$.

The deregulation of ING genes was frequently detected in various types of cancer $(9,11)$. ING1 gene expression was observed to be downregulated or lost in various types of cancer including breast, gastric, esophageal, lung and brain (2,12-16). Previous studies investigating ING2 in cancer have suggested that a reduction in expression is involved in the initiation of melanoma and the progression of hepatocellular carcinoma (HCC) $(17,18)$. Certain studies have reported that ING4 expression was significantly suppressed in brain tumors, HCC, breast cancer and head and neck squamous cell carcinoma (HNSCC) (16,19-21). Our previous study also reported the decreased expression of ING5 gene in HCC (22). Therefore, the deregulation of ING genes may contribute to tumorigenesis (8).

The ING3 gene, which encodes a $46.8 \mathrm{kDa}$ protein, has been associated with the modulation of p53-mediated transcription, cell cycle control and apoptosis (5). Findings of previous studies demonstrated that the ectopic expression of ING3 in RKO cells decreased colony formation and the number of cells in the $\mathrm{S}$ phase. Although physical association with $\mathrm{p} 53$ is required for the function of the other ING members, ING3 does not appear to interact with p53 (8). In melanoma cells, ING3 overexpression promotes UV-induced apoptosis through a Fas/caspase-8-dependent pathway in a p53-independent manner (23). ING3 has been reported to be a tumor suppressor in melanoma and HNSCC $(24,25)$. Low levels of ING3 mRNA may indicate an aggressive head and neck carcinoma. In melanoma, ING3 nuclear expression is reduced and may be an independent prognostic factor (21).

In this study, the expression of ING3 was evaluated in tissues at different stages of $\mathrm{HCC}$ using the reverse transcription-polymerase chain reaction (RT-PCR) and an immunohistochemical assay of tissue microarray (TMA). ING3 was significantly downregulated in malignant HCC tissue. Moreover, it was demonstrated that ING3 suppressed HCC cell proliferation, colony formation and inhibited cell migration. This suggests that the deregulation of ING3 is involved in the tumorigenesis and metastasis of HCC.

\section{Materials and methods}

Tissue specimens and cell lines. The tumor and normal liver specimens were obtained from patients who had provided 
informed consent. HepG2, Hep3B, Huh7, Bel-7402, Bel-7404, Bel-7405, PLC, PCL/PRF/5, LM3, LM6, QCY-7701, SNU398, MHCC-H, MHCC-L, YY-8103, SK-HEP, SMMC-7721 and Focus were the 18 liver tumor-derived cell lines used in this study. The study and the protocol for the use of human tissues for this study were approved by the ethics committee of the Chinese National Human Genome Center (Shanghai, China).

Plasmids and antibodies. The entire open reading frame of human ING3 was subcloned into pcDNA3.0 (Invitrogen, Carlsbad, CA, USA) mammalian cell expression vectors. pGEX5x-1-ING3 was constructed to produce the GST-ING3 fusion protein for generating antibodies against human ING3. Rabbit polyclonal anti-ING3 antibodies were raised against the GST-ING3 fusion protein and purified from anti-serum with protein G sepharose beads (Roche Diagnostics, Mannheim, Germany). The specificity of the ING3 antibody was verified by western blot analysis with the protein samples from the cells transfected with plasmids expressing ING1-5 (data not shown). Mouse anti-actin antibody was purchased from Sigma (St. Louis, MO, USA).

$R N A$ extraction and real-time RT-PCR. Total RNA was extracted using TRIzol solution (Invitrogen) in accordance with the manufacturer's instructions. Reverse transcription was performed in a $20 \mu 1$ reaction system with $2 \mu \mathrm{g}$ total RNA treated with M-MLV reverse transcriptase to synthesis first-strand cDNA (Promega, Madison, WI, USA). Real-time quantitative RT-PCR was performed with specific primers for ING3 and GAPDH served as an internal control. The sequences of the sense and antisense primers were as follows: ING3: 5'-ACCTGAGTGGAGGGAAGAGC-3' (F) and 5'-CTGGTTTGCCAACTGAACCT-3' (R); GAPDH: 5'-GAAGGTGAAGGTCGGAGTC-3' (F) and 5'-GAAGATG GTGATGGGATTTC-3' (R).

Immunohistochemical analysis. Slides containing $121 \mathrm{HCC}$ specimens with adjacent noncancerous tissues (Shanghai Biochip Company Ltd., Shanghai, China) were used to evaluate ING3 expression via immunohistochemistry. The slides were incubated overnight at $4^{\circ} \mathrm{C}$ with rabbit anti-ING3 polyclonal antibody (1:200 dilution), followed by incubation with a horseradish peroxidase-conjugated anti-rabbit secondary antibody (Dako Japan Ltd., Kyoto, Japan) at $37^{\circ} \mathrm{C}$ for $30 \mathrm{~min}$. Normal rabbit IgG was used as a negative control. The signals were visualized using 3,3'-diaminobenzidine tetrahydrochloride (DAB). The slides were counterstained with hematoxylin, dehydrated through gradient alcohols and mounted for observation. The total ING3 immunostaining was calculated as the sum of the relative positivity of stained tumor cells and the staining intensity. The relative positivity was scored as weakly, moderately and strongly stained. The final ING3 expression levels were defined as: 'upregulated', 'equal' and 'downregulated' in HCC samples compared with noncancerous livers.

Cell culture and transfection. Cell lines were cultured in DMEM-high supplemented with $10 \%$ fetal bovine serum (Gibco, Carlsbad, CA, USA) in a $5 \% \mathrm{CO}_{2}$ atmosphere at $37^{\circ} \mathrm{C}$. The expression vector pcDNA3.0 and the pcDNA3.0-ING3 plasmids were transfected by lipofectamine 2000 (Invitrogen) according to the manufacturer's instructions.

Cell proliferation and colony formation. To observe cell proliferation, the Hep3B cells transfected with ING3 were seeded in 96-well plates at 3,000 cells/well and cultured for 6 days. Cell viability was measured using the Cell Counting Kit-8 (Dojindo Laboratories, Kunamoto, Japan). HCC cells transfected with pcDNA3.0-ING3 were cultured on $100-\mathrm{mm}$ plates and selected with G418 (Life Technologies, Inc., Carlsbad, CA, USA) at a final concentration of 0.6 to $1 \mathrm{mg} / \mathrm{ml}$ for colony formation.

Wound-healing assay. The transfected cells were plated in 60-mm dishes at the same density, scratch wounded with a micropipette tip, washed to remove detached cells, provided with fresh medium including $2 \%$ FBS and were incubated at $37^{\circ} \mathrm{C}$ during image capture. The wounds were marked under the dish with a felt tip pen and images were captured at these sites using a x10 objective at $0 \mathrm{~h}$ and again at 24 and $48 \mathrm{~h}$. Cell motility was evaluated using the formula: Wound closure $=$ (distance $_{24 \text { or } 48 \mathrm{~h}}-$ distance $_{0 \mathrm{~h}}$ )/ distance $_{0 \mathrm{~h}}$.

Western blot analysis. Following transfection, the cells were obtained via lysis buffer [25 mmol/1 Tris (pH 6.8), 1\% SDS, $5 \mathrm{mmol} / \mathrm{l}$ EDTA, protease inhibitor cocktail (Sigma)]. The protein samples were resolved via SDS-PAGE and transferred onto a nitrocellulose membrane which was blocked in 5\% skimmed milk in phosphate-buffered saline (PBS) containing Tween (PBS-Tween) and probed with the indicated antibodies. The membrane was scanned on an Odyssey infrared imaging system (LI-COR) at a wavelength of 700 or $800 \mathrm{~nm}$. Rabbit anti-ING3 and mouse anti- $\beta$-actin (Sigma) antibodies were used in this study.

Statistical analysis. Quantitative values are shown as the mean \pm standard deviation (SD) or median (range). A paired samples t-test was used to determine the difference between $\mathrm{HCC}$ and noncancerous livers. A $\chi^{2}$ test was performed to analyze the correlation between ING3 expression and various clinicopathological characteristics. The SPSS (Chicago, IL, USA) 13.0 software was used for all statistical analyses. $\mathrm{P}<0.05$ was considered to indicate a statistically significant result.

\section{Results}

Decreased expression of ING3 in HCC. Previous studies have suggested that most members of the ING family, including ING1, ING2, ING4 and ING5, are downregulated in HCC $(16,18,26,27)$. In the present study, the relative expression levels of ING1-5 were investigated in 18 pairs of human HCC specimens by real-time RT-PCR and it was observed that the mRNA levels of the five ING family members were significantly downregulated in HCC tumor tissues (22). To understand the correlation of ING3 expression with HCC, the mRNA expression level of ING3 in an additional 31 human HCC specimens was observed. ING3 was markedly downregulated in all 49 tumor tissues compared with adjacent noncancerous livers (Fig. 1A). The semiquantitative RT-PCR of 3 pairs of randomly selected human HCC specimens confirmed the observation of a decreased ING3 expression in HCC tissues 
A

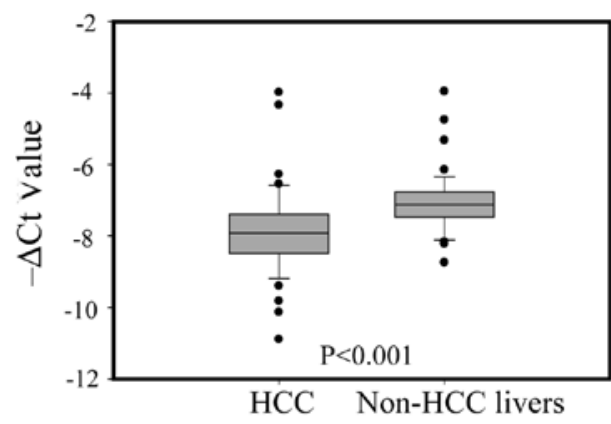

B

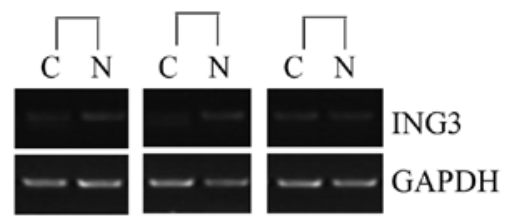

C

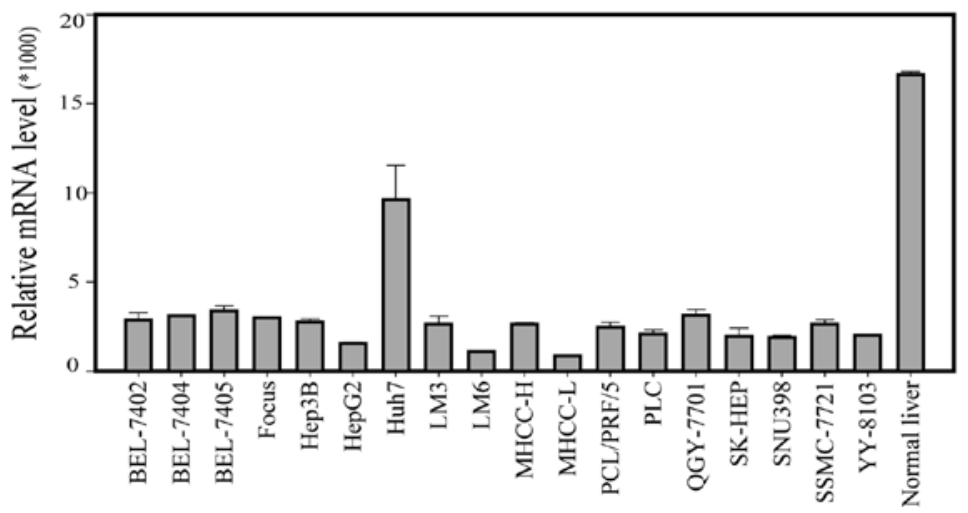

Figure 1. Expression pattern of ING3 in HCC samples and cell lines. (A) The transcript level of ING3 was measured in 49 paired HCC and adjacent non-cancerous livers by quantitative RT-PCR, where GAPDH was used as an internal reference. The line within each box is the median $-\Delta \mathrm{Ct}$ value; the upper and lower edges of each box show the 75th and 25th percentile, respectively. The upper and lower bars are the highest and lowest values. P-value was calculated by paired sample t-test $(\mathrm{p}<0.001)$. (B) Representative semi-quantitative RT-PCR of ING3 of 3 pairs of HCC samples. C, cancer tissue; N, adjacent noncancerous liver. (C) Relative mRNA level of ING3 was evaluated in HCC cell lines and fetal liver by semiquantitative RT-PCR. HCC, hepatocellular carcinoma; ING3, inhibitor of growth 3; RT-PCR, reverse transcription-polymerase chain reaction.
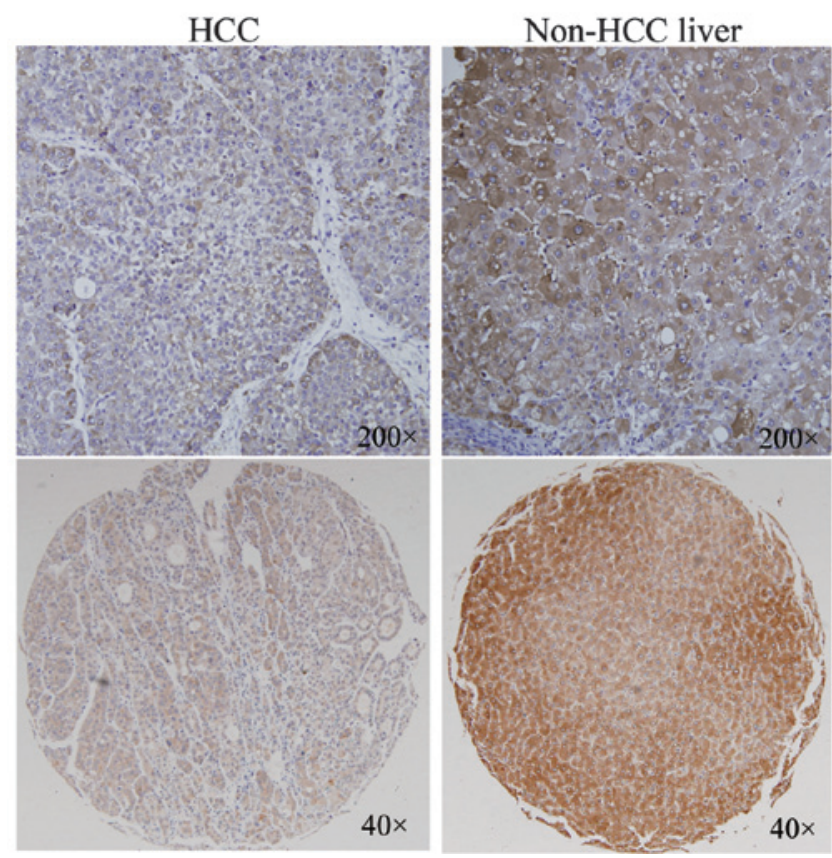

Figure 2. Representative immunohistochemical detection of the ING3 protein expression in HCC and surrounding noncancerous tissues. HCC, hepatocellular carcinoma; ING3, inhibitor of growth 3.

(Fig. 1B). The corresponding mRNA expression in 18 typical HCC cell lines was also examined by RT-PCR (Fig. 1C). The cultured HCC cells expressed various levels of ING3, which were lower than those of normal liver tissue.
Immunohistochemistry and clinicopathological analysis. To investigate the ING3 protein expression in human HCC tissues, a tissue array containing 112 pairs of HCC specimens was examined by immunohistochemical staining with an ING3 antibody (Fig. 2). The expression of the ING3 protein was downregulated in 57.14\% (64/112) HCC tissues compared with the corresponding noncancerous livers, while the upregulation of ING3 was detected in $21.42 \%$ (24/112) samples of $\mathrm{HCC}$ and the remaining $24 \mathrm{HCC}$ tissues exhibited almost the same level of ING3 expression (Table I). To understand the correlation of ING3 levels with clinicopathological features, the $\chi^{2}$ test was used to analyze the correlations between the staining intensity of the ING3 protein and the clinicopathological variables of HCC (Table I). The ING3 protein staining intensity showed no significant correlation with gender or age ( $>0.05$; Table I). However, the downregulation ratio of the ING3 protein was significantly greater in the HCC samples with Edmondson-Steiner grades II/III than in those with Edmondson-Steiner grades I/II (Table I; p=0.004), suggesting that a low expression level of ING3 in HCC is associated with tumor differentiation and classification.

ING3 inhibits cell proliferation and colony formation. To investigate the effect of ING3 on HCC cells in vitro, recombinant pcDNA3.0-ING3 plasmids were used to induce the transient overexpression of ING3 in hepatoma cells. The expression level of ING3 in Hep3B and Huh7 cells transfected with the indicated plasmids was detected with western blot analysis (Fig. 3A). The tumor cell growth was examined daily for 6 days using the CCK- 8 assay and the ability to form 
Table I. Correlations between ING3 expression and clinicopathological variables of 112 cases of HCC.

\begin{tabular}{|c|c|c|c|c|c|c|}
\hline \multirow{2}{*}{$\begin{array}{l}\text { Clinicopathological } \\
\text { variables }\end{array}$} & \multirow{2}{*}{$\begin{array}{l}\text { Number of } \\
\text { patients }\end{array}$} & \multicolumn{3}{|c|}{ ING3 expression level $\left(\mathrm{C}^{\mathrm{a}}\right.$ compared with $\left.\mathrm{N}^{\mathrm{b}}\right)$} & \multirow[b]{2}{*}{ P-value } & \multirow[b]{2}{*}{$\chi^{2}$} \\
\hline & & Upregulated n (\%) & Equal n (\%) & Downregulated n (\%) & & \\
\hline All cases & 112 & $24(21.42)$ & $24(21.42)$ & $64(57.14)$ & & \\
\hline Age (years) & & & & & 0.182 & 3.412 \\
\hline$\geq 50$ & 79 & $20(25.32)$ & $18(22.78)$ & $41(51.90)$ & & \\
\hline$<50$ & 33 & $4(12.12)$ & $6(18.18)$ & $23(69.70)$ & & \\
\hline Gender & & & & & 0.982 & 0.037 \\
\hline Male & 102 & $22(21.57)$ & $22(22.57)$ & $58(56.86)$ & & \\
\hline Female & 10 & $2(20.00)$ & $2(20.00)$ & $6(60.00)$ & & \\
\hline Edmondson-Steiner grade & & & & & $0.004^{c}$ & 11.000 \\
\hline Low I/II & 28 & $12(42.85)$ & $6(21.43)$ & $10(35.71)$ & & \\
\hline High II/III & 84 & $12(14.29)$ & $18(21.43)$ & 54 (64.29) & & \\
\hline
\end{tabular}

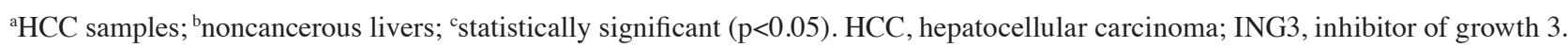

A
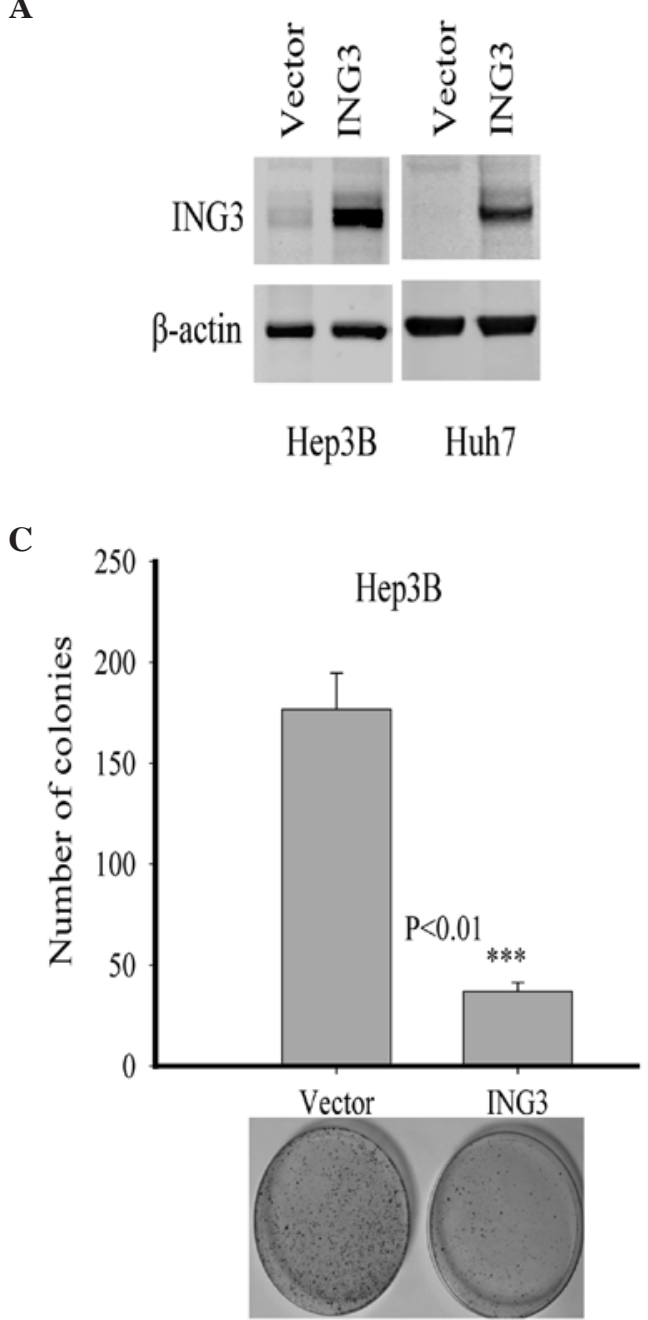

B

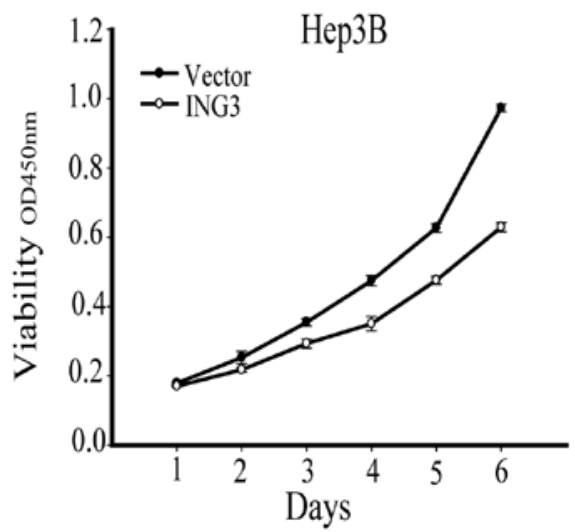

D

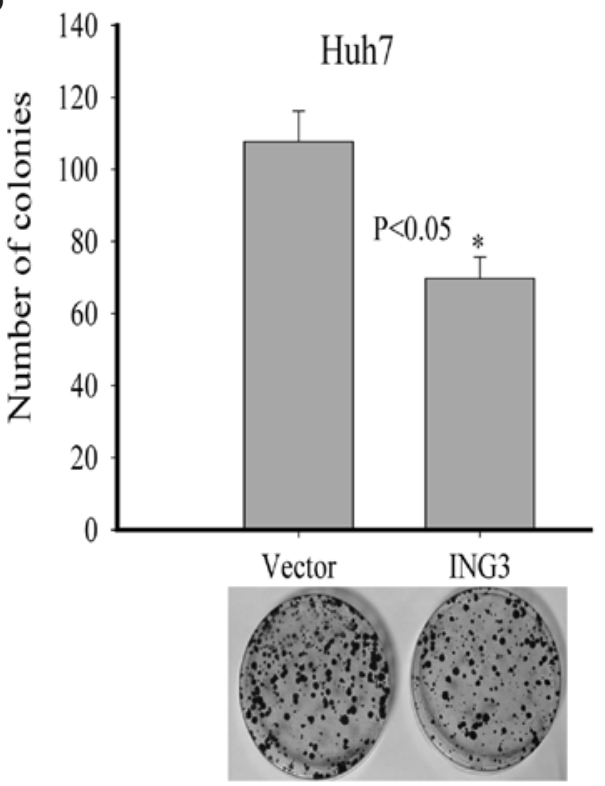

Figure 3. ING3 overexpression inhibits cell proliferation and colony formation. (A) Exogenous ING3 was expressed in Hep3B and Huh7 cells by transient transfection with pcDNA3.0-ING3, confirmed by western blot analysis. Cells transfected with an empty vector were used as the control. (B) The growth curve of Hep3B cells with the exogenous ING3. Cells transfected with the empty vector pcDNA3.0 served as controls. The experiments were repeated three times and the spots represent the average values of triplicate wells, with SD included for each mean value. (C) ING3 suppressed the ability of Hep3B cells to form colonies. The relative numbers of colonies of Hep3B cells transfected with pcDNA3.0-ING3 and a control vector were calculated from three independent experiments $\left({ }^{* * *} \mathrm{p}<0.01\right)$. The lower panel showed the representative dishes of the experiments. (D) ING3 inhibited the colony formation of Huh7 cells $\left({ }^{*} \mathrm{p}<0.05\right.$ as compared with the control vectors). ING3, inhibitor of growth 3 . 
A

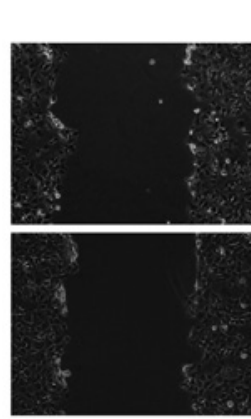

$0 \mathrm{~h}$

B

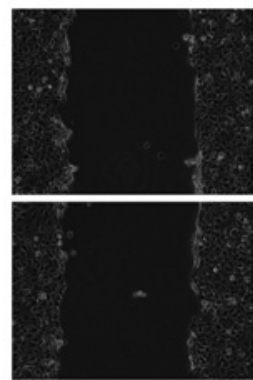

$0 \mathrm{~h}$
Hep3B

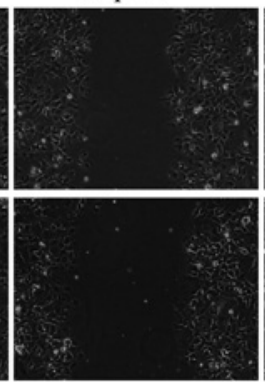

$24 \mathrm{~h}$

Huh7

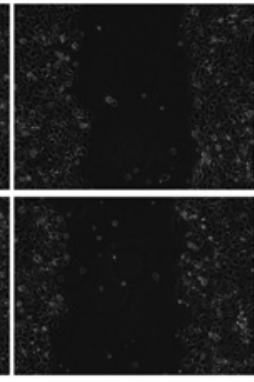

$24 \mathrm{~h}$

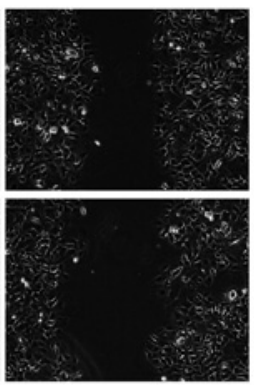

$48 \mathrm{~h}$

Vector

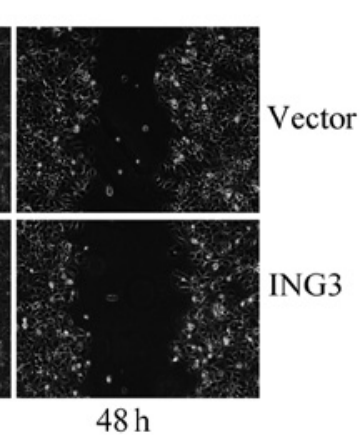

C

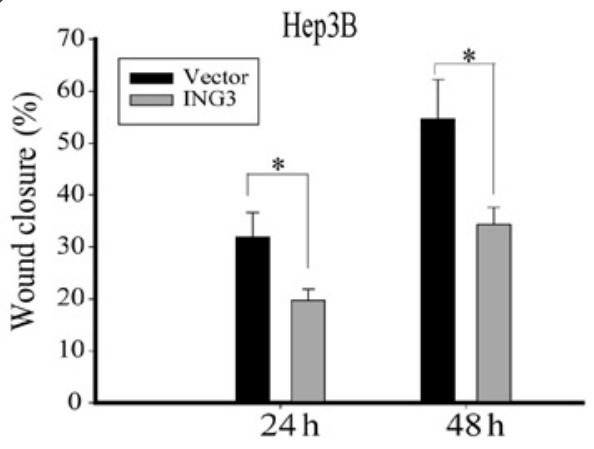

D

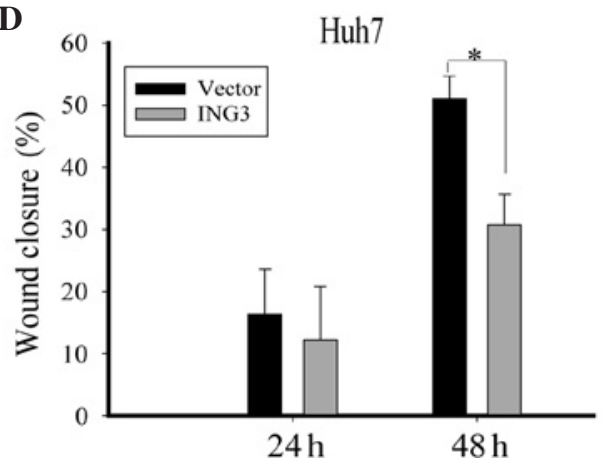

Figure 4. ING3 inhibits the migration of hepatoma cells. (A and B) ING3 overexpression reduced the migration of (A) Hep3B and (B) Huh7 cells. The two cell types were scraped with micropipette tips following transfection with pcDNA3.0-ING3 or pcDNA3.0. Images were captured at 0,24 and 48 h following wounding. Cells transfected with an empty vector were used as controls. (C and D) The wound healing rates at 24 and $48 \mathrm{~h}$ following wounding of (C) Hep3B and (D) Huh7 cells. Values are the mean \pm SD. ${ }^{*}$ p $<0.05$. ING3, inhibitor of growth 3.

colonies was measured following the treatment of the cells with G418. As shown in Fig. 3B, the overexpression of ING3 significantly suppressed cell proliferation of Hep3B cells $(\mathrm{p}<0.05)$. In addition, the colony formation was also markedly inhibited by ING3 in Hep3B cells (Fig. 3C; p<0.01). In Huh7 cells, although no effect on the cell proliferation was detected (data not shown), ING3 greatly decreased the colony formation (Fig. 3D; $\mathrm{p}<0.05$ ). These data suggest that as a tumor suppressor, ING3 inhibited the hepatoma cell growth in vitro.

ING4 inhibits hepatoma cell migration. Cell motility plays a significant role during tumor progression and tumor metastasis. The present study also showed that the downregulation of ING3 was correlated with the grade of HCC. The effect of ING3 on hepatoma cell migration was then investigated using the wound-healing assay. The Hep3B and Huh7 cells with ING3 overexpression were scratched and monitored for wound healing with time. Notably, the hepatoma cells in which ING3 was overexpressed migrated markedly slower than the control cells (Fig. 4A and B). The wound healing rate of the Hep3B and Huh7 cells was significantly decreased by ING3 overexpression (Fig. 4C and D, p<0.05). These data indicate that ING3 impaired the migration ability of hepatoma cells, implying that ING3 plays an inhibitory role in HCC metastasis.

\section{Discussion}

The ING family members are type II tumor suppressor genes and have been reported to be involved in multiple cell processes, including cell growth and proliferation, senescence, DNA repair, oncogenesis, apoptosis, angiogenesis and tumorigen- esis $(8,9)$. Previous studies have indicated that the ING family is involved in HCC pathogenesis. The expression of ING1 variants was markedly reduced in HCC samples. ING1 inhibited the hepatoma cell growth via stabilization and activation of p53 by interacting with Mdm2 and p14ARF $(26,27)$. As a candidate tumor suppressor gene, ING2 was involved in the progression of HCC (18). The downregulation of ING2 has been observed in $52.8 \%$ of HCC and is associated with tumor size, histopathological classification and serum $\alpha$-fetoprotein. Similarly, ING4 expression was found to be significantly reduced in $\mathrm{HCC}$ and the expression level also correlated with patient prognosis and the metastatic potential of HCC (16). In a recent study, we reported that the suppression of ING5 was correlated with the progression of HCC (22). However, the role of ING3 in human HCC remains unknown. In this study, the mRNA expression of ING3 in 49 paired HCC samples and 18 HCC cell lines was investigated by RT-PCR. The results of this study showed that approximately $83.67 \%$ (33/49) HCC samples expressed lower levels of ING3 mRNA than the adjacent noncancerous tissues and the expression of ING3 was decreased in the HCC cell lines. Immunohistochemical staining of the tissue array consistently indicated that the ING3 protein was also downregulated in HCC compared with the corresponding noncancerous liver specimens. Notably, the downregulation of ING3 was more frequent in moderate and high Edmondson-Steiner grades of tumor. Previous studies have reported that Edmondson-Steiner grades were closely associated with tumor progression and differentiation. Therefore, the decreased ING3 expression may be associated with tumor progression.

A previous study has shown that ING3 regulated the cell cycle and apoptosis. Nagashima et al indicated that ING3 over- 
expression may reduce the number of RKO cells in S phase and induce p53-dependent apoptosis (5). Wang et al noted that ING3 significantly promoted UV-induced apoptosis through the activation of the Fas/caspase-8 pathway in melanoma cells (23). In the present study, cell proliferation, colony formation and wound-healing assays were performed to investigate the functional role of ING3 in HCC cells. The ectopic expression of ING3 in Hep3B and Huh7 cells inhibited colony formation and cell migration significantly, suggesting that ING3 is involved in tumorigenesis and metastasis. These findings were consistent with the immunohistochemical staining and tissue array results. However, the cell proliferation results of these HCC cells demonstrated a different effect of ING3. Overexpression of ING3 suppressed the cellular growth in Hep3B cells but not in Huh7 cells, suggesting that the cellular inhibitory effect of ING3 in HCC depends on various mechanisms.

Previous studies have suggested that ING3 was downregulated in HSNCC and may play a suppressing role in melanoma tumorigenesis. The degradation of ING3 protein by SCF-mediated ubiquitin-proteasome system in melanoma results in progression of the tumor (28). Another study showed that the nuclear-to-cytoplasmic translocation of ING3 resulted in a decreased nuclear expression and was involved in melanoma initiation and tumor progression (25). However, the potential role of ING3 as a tumor suppressor in other types of cancer remains unclear. In this study, downregulation of ING3 was found to be correlated with tumorigenesis and the progression of HCC. Moreover, ING3 may inhibit the tumorigenesis through suppressing cell proliferation and colony formation. Furthermore, ING3 may impede tumor metastasis by inhibiting cell migration. The role played by the molecular mechanism of ING3 in the suppression of HCC requires further clarification; however, the present study indicated that ING3 is a potential therapeutic target of HCC. The function of ING3 in HCC tumorigenesis and progression therefore merits further investigation.

\section{Acknowledgements}

The study was supported by Chinese High-Tech Research and Development Program Grants (2006AA02A305), the National Natural Science Foundation of China (81071842) and the Shanghai Commission for Science and Technology (10JC1411900).

\section{References}

1. He GH, Helbing CC, Wagner MJ, Sensen CW and Riabowol K: Phylogenetic analysis of the ING family of PHD finger proteins. Mol Biol Evol 22: 104-116, 2005.

2. Toyama T, Iwase H, Watson P, et al: Suppression of ING1 expression in sporadic breast cancer. Oncogene 18: 5187-5193, 1999.

3. Nagashima M, Shiseki M, Miura K, et al: DNA damage-inducible gene p33ING2 negatively regulates cell proliferation through acetylation of p53. Proc Natl Acad Sci USA 98: 9671-9676, 2001.

4. Shimada Y, Saito A, Suzuki M, Takahashi E and Horie M: Cloning of a novel gene (ING1L) homologous to ING1, a candidate tumor suppressor. Cytogenet Cell Genet 83: 232-235, 1998.
5. Nagashima M, Shiseki M, Pedeux RM, et al: A novel PHD-finger motif protein, p47ING3, modulates p53-mediated transcription, cell cycle control, and apoptosis. Oncogene 22: 343-350, 2003.

6. Shiseki M, Nagashima M, Pedeux RM, et al: p29ING4 and p28ING5 bind to 533 and p300, and enhance p53 activity. Cancer Res 63: 2373-2378, 2003.

7. Zhang X, Xu LS, Wang ZQ, et al: ING4 induces G2/M cell cycle arrest and enhances the chemosensitivity to DNA-damage agents in HepG2 cells. FEBS Lett 570: 7-12, 2004.

8. Coles AH and Jones SN: The ING gene family in the regulation of cell growth and tumorigenesis. J Cell Physiol 218: 45-57, 2009.

9. Gong W, Suzuki K, Russell M and Riabowol K: Function of the ING family of PHD proteins in cancer. Int J Biochem Cell Biol 37: 1054-1065, 2005.

10. Russell M, Berardi P, Gong W and Riabowol K: Grow-ING, Age-ING and Die-ING: ING proteins link cancer, senescence and apoptosis. Exp Cell Res 312: 951-961, 2006.

11. Campos EI, Chin MY, Kuo WH and Li G: Biological functions of the ING family tumor suppressors. Cell Mol Life Sci 61: 2597-2613, 2004

12. Tokunaga E, Maehara Y, Oki E, et al: Diminished expression of ING1 mRNA and the correlation with p53 expression in breast cancers. Cancer Lett 152: 15-22, 2000.

13. Oki E, Maehara Y, Tokunaga E, Kakeji Y and Sugimachi K: Reduced expression of p33(ING1) and the relationship with p53 expression in human gastric cancer. Cancer Lett 147: 157-162, 1999.

14. Hara Y,Zheng Z, Evans SC, et al: ING1 and p53 tumor suppressor gene alterations in adenocarcinomas of the esophagogastric junction. Cancer Lett 192: 109-116, 2003.

15. Okano T, Gemma A, Hosoya Y, et al: Alterations in novel candidate tumor suppressor genes, ING1 and ING2 in human lung cancer. Oncol Rep 15: 545-549, 2006.

16. Fang F, Luo LB, Tao YM, Wu F and Yang LY: Decreased expression of inhibitor of growth 4 correlated with poor prognosis of hepatocellular carcinoma. Cancer Epidemiol Biomarkers Prev 18: 409-416, 2009.

17. Lu F, Dai DL, Martinka M, Ho V and Li G: Nuclear ING2 expression is reduced in human cutaneous melanomas. Br J Cancer 95: 80-86, 2006.

18. Zhang HK, Pan K, Wang H, et al: Decreased expression of ING2 gene and its clinicopathological significance in hepatocellular carcinoma. Cancer Lett 261: 183-192, 2008.

19. Garkavtsev I, Kozin SV, Chernova O, et al: The candidate tumour suppressor protein ING4 regulates brain tumour growth and angiogenesis. Nature 428: 328-332, 2004.

20. Kim S, Chin K, Gray JW and Bishop JM: A screen for genes that suppress loss of contact inhibition: identification of ING4 as a candidate tumor suppressor gene in human cancer. Proc Natl Acad Sci USA 101: 16251-16256, 2004.

21. Gunduz M, Nagatsuka H, Demircan K, et al: Frequent deletion and down-regulation of ING4, a candidate tumor suppressor gene at $12 \mathrm{p} 13$, in head and neck squamous cell carcinomas. Gene 356: 109-117, 2005.

22. Lu ML, Chen F, Wang QW, Han ZG and Zhang X: Study on expression of ING5 in hepatocellular carcinoma. Wei Chang Bing Xue Za Zhi Tou Gao Xu Zhi 15: 86-89, 2010.

23. Wang Y and Li G: ING3 promotes UV-induced apoptosis via Fas/caspase-8 pathway in melanoma cells. J Biol Chem 281: 11887-11893, 2006.

24. Gunduz M, Beder LB, Gunduz E, et al: Downregulation of ING3 mRNA expression predicts poor prognosis in head and neck cancer. Cancer Sci 99: 531-538, 2008.

25. Wang Y, Dai DL, Martinka M and Li G: Prognostic significance of nuclear ING3 expression in human cutaneous melanoma. Clin Cancer Res 13: 4111-4116, 2007.

26. Ohgi T, Masaki T, Nakai S, et al: Expression of p33(ING1) in hepatocellular carcinoma: relationships to tumour differentiation and cyclin E kinase activity. Scand J Gastroenterol 37: 1440-1448, 2002.

27. Zhu Z, Luo Z, Li Y, Ni C, Li H and Zhu M: Human inhibitor of growth 1 inhibits hepatoma cell growth and influences p 53 stability in a variant-dependent manner. Hepatology 49: 504-512, 2009.

28. Chen G, Wang Y, Garate M, Zhou J and Li G: The tumor suppressor ING3 is degraded by SCF(Skp2)-mediated ubiquitinproteasome system. Oncogene 29: 1498-1508, 2010. 